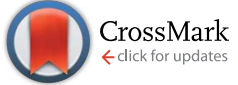

Cite this: RSC Adv., 2015, 5, 85039

\title{
CIELab chromaticity evolution to measure the binding free energy of non-colored biomolecules to gold nanoparticles $\dagger$
}

\author{
R. Prado-Gotor, ${ }^{\star}$ A. Jimenez-Ruiz, ${ }^{*}$ J. M. Carnerero, E. Grueso and I. Villa
}

We explore the possibilities of the CIELab chromaticity system parameters' evolution in order to study the interaction between two non-colored biological reactants, lysozyme and adenosine, and the red-colored, non-functionalized citrate gold nanoparticles. By measuring and quantifying the AuNP color changes, which are strongly dependant on their aggregation state, binding free energies are obtained for the first time for these two systems. Results are confirmed via an alternate fitting method which makes use of deconvolution parameters from experimental absorbance spectra. Binding free energies obtained through the use of both means are in good agreement with each other.

Received 29th July 2015

Accepted 1st October 2015

DOI: $10.1039 / \mathrm{c} 5 \mathrm{ra15119h}$

www.rsc.org/advances

The CIELab color system is a standard on colorimetry, based

\section{Introduction}

Gold nanoparticles (AuNPs) present excellent biocompatibility with biomolecules and display promising applications because of their structural, electronic, magnetic, optical and catalytic properties, which are very different from those of the corresponding bulk materials. Biomaterials such as proteins, enzymes or DNA strands and bases are known to display highly selective catalytic and recognition properties, a fact that makes them important assets in biosensor development studies..$^{1-4}$ From both types of materials a very active research area has developed in order to study these AuNP-biomolecule hybrids.

As for the nature of the interactions between the nanoparticle and the biomolecule, chemisorptions, electrostatic adsorptions, covalent binding or specific affinity interactions may be present with more or less force, making binding energy quantification in these systems extremely important. In order to determine the AuNPs-biomolecule equilibrium binding constant, either (a) a suitable, easy-to-follow property of the interacting biomolecule, or (b) in the case of functionalized AuNPs, a similarly appropriate property of the capping ligand needs to be chosen. But, what if AuNPs are not functionalized or the interaction with the biomolecule cannot be followed by any simple means, for example, fluorometric or spectrophotometric techniques? Our study aims to explore if the CIELab uniform color space parameters $\mathrm{a}^{*}$ and $\mathrm{b}^{*}$ can be successfully applied to quantify the binding interactions in these cases.

Department of Physical Chemistry, Faculty of Chemistry, University of Sevilla, C/ Profesor García González s/n, 41012 Sevilla, Spain. E-mail: pradogotor@us.es; ailjimrui@alum.us.es

$\dagger$ Electronic supplementary information (ESI) available. See DOI: 10.1039/c5ra15119h on the tristimulus theory of vision, which aspires to mimic human vision by computing as "opposite" tones that come from inverse stimuli on the eye, and therefore cannot be perceived at the same time; those opposite colors are red to green, and blue to yellow. On a CIELab space, three coordinates are used to fully describe a given color: $\mathrm{L}^{*}$, which can be identified as the "lightness" of the tone on a scale from 0 (black) to 100 (white), $\mathrm{a}^{*}$, which measures the green-versus-red component of the tone, and $\mathrm{b}^{*}$, for the yellow-versus-blue component. CIELab coordinates have been extensively used for color kinetic analysis in applied chemistry fields, such as food technology $y^{5-7}$ or material sciences. ${ }^{8,9}$ Still, those colorimetric parameters have never been applied to the analysis and determination of binding constants between a colored nanoparticle and a non-colored reactant. It is important to note that regardless of the selectivity or accuracy of the chosen detection method, or detection being done with functionalized or non-functionalized gold nanoparticles, CIELab parameters can offer an hindsight into the aggregationdisaggregation processes taking place in solution; we propose that these data can then be employed to determine binding parameters, and as such, the AuNPs/lysozyme and AuNPs/ adenosine systems in water have been chosen as representative examples of colorimetric parameters' application to an experimental system. The former presents well-known fluorescence properties that can offer an additional hindsight into the processes being studied, and the latter is a colorless, nonfluorescent molecule lacking any easy-to-follow properties that could allow for binding parameters determination.

Lysozyme (Lys) is a model protein whose structure and properties are already well known; one of its most interesting features is the way changes of lysozyme levels in body fluids have been reported to be associated with various illnesses. For example, increased Lys concentration in urine and serum is 
associated with leukemia ${ }^{\mathbf{1 0 , 1 1}}$ renal diseases ${ }^{\mathbf{1 2}}$ and meningitis, ${ }^{\mathbf{1 3}}$ a fact that makes fast and reliable detection and quantification methods for the protein extremely important. It also exhibits the ability to electrostatically bind human serum albumin (HSA) and other negatively charged proteins. ${ }^{\mathbf{1 4 , 1 5}}$ On the other hand, the interaction of metal nanoparticles and surfaces with DNA bases and nucleotides is a relevant topic which has been the subject of extensive research in recent years; ${ }^{16-18}$ for example, it was recently studied by using DFT and post Hartree-Fock calculations. ${ }^{19}$ Nevertheless, even though the affinity order of both nucleosides and DNA bases to gold has already been determined by several methods $\mathbf{s}^{16,18,20,21}$ to our knowledge no studies have offered a quantitative determination of the binding free energies of nucleosides to AuNPs. In order to test this approach of our colorimetric method, we have chosen adenosine (Ad) as a model nucleoside. Unlike lysozyme, adenosine is not fluorescent at neutral/basic pH. Fluorescence measurements have been reported for adenosine at strongly acidic $\mathrm{pH},{ }^{22,23}$ but this emission has been identified as coming from a protonated tautomer whose proportion at pH 6 is already minimal.

In order to confirm the robustness of the method, two additional non-fluorescent biomolecules of a different nature were used. The first one was L-lysine, a proteinogenic amino acid with an additional $-\mathrm{NH}_{3}{ }^{+}$residue in its lateral chain. The second one was thiourea, whose interest as a biomolecule that can interact with, and cause aggregation of, gold nanoparticles has been recently proven with a study in which its detection is carried out by using naked-eye colorimetric techniques. ${ }^{24}$ Data for those biomolecules can be found in the ESI. $\dagger$

\section{Results and discussion}

Fig. 1 and $\mathrm{S} 2 \dagger$ show the experimental surface plasmon resonance band (SPR) maximum shift corresponding to $17 \mathrm{~nm}$ citrate gold nanoparticles in the presence of different biomolecule concentrations. $\lambda_{\max }$ changes to higher values in the presence of either lysozyme or adenosine, and this shift of the absorption band causes a noticeable variation in the color of the solutions (from the characteristic red tint of gold nanoparticles to blue) that can be followed even at naked eye. In the case of lysozyme, the $\Delta \lambda$ plot versus [Lys] presents a maximum; this is due to the SPR of aggregated nanoparticles shifting to lower wavelengths at the highest lysozyme concentrations. This effect can be attributed to nanoparticle aggregation reverting due to the positively charged protein interacting with the AuNPs in lieu of the citrate anions, which the protein replaces; it is also interesting to note that in addition to their red color, nanoparticles on solutions that also contain high lysozyme concentrations also show increased stability when compared to their citrate counterparts. For adenosine, no further changes are observed once $\Delta \lambda=12 \mathrm{~nm}\left([\mathrm{Ad}]=4 \times 10^{-4} \mathrm{M}\right)$, and higher adenosine concentrations do not cause this value to shift.

Fig. 2 and $\mathrm{S} 3 \dagger$ shows the color changes of nanoparticle solutions, reflected on shifts of $\mathrm{a}^{*}$ and $\mathrm{b}^{*}$ parameters, when either lysozyme or adenosine concentration increases. As can be seen, $b^{*}$ values shift from positive to negative at low concentrations, which indicates a yellow to blue shift on the tone of the
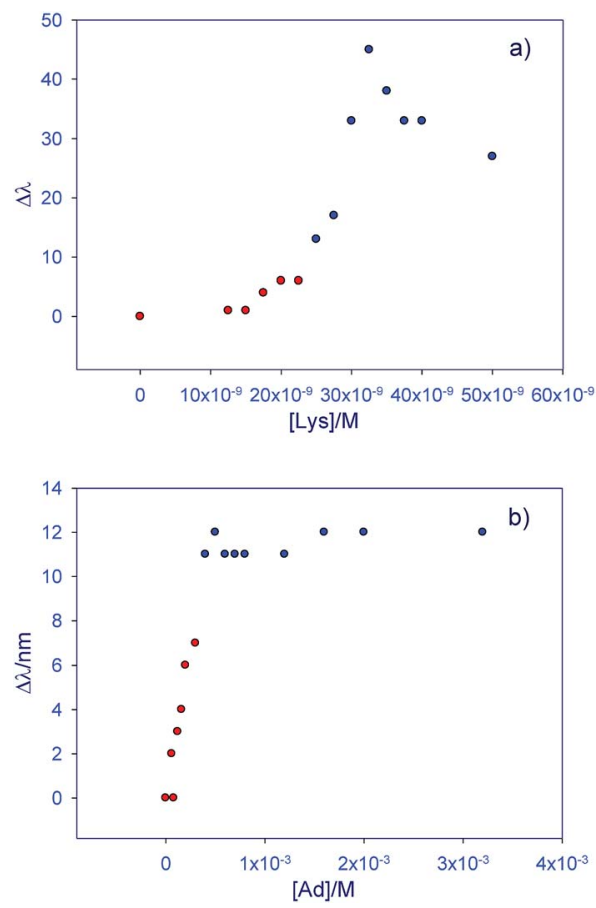

Fig. 1 Wavelength shift (expressed as $\Delta \lambda=\lambda_{\text {AunPs(free) }}-$ $\left.\lambda_{\text {AuNPs(aggregated) }}\right)$ of the most intense experimental absorbance peak for a series of AuNPs solutions containing (a) lysozyme and (b) adenosine. Solutions where $\Delta \lambda<10$ show a red color and are marked as such in the graph.

solution. This fact accounts for the appearance of the blue tint from aggregated nanoparticles. At the same time, a* values shift from more to less positive values with increasing [Lys] or [Ad], as the aggregates form and the red color from free nanoparticles starts to fade. ${ }^{*}$ values not becoming negative (that is, not fully shifting from red to green) in neither case is an indication of the final color of the aggregated solutions being purple and not fully blue, a fact that accurately reflects the experimental data. For high [Lys], a* values grow once again, as the solutions start to shift back from purple to red due to the aforementioned disaggregation by lysozyme protection. Notice that the previously reported maximum observed for $\Delta \lambda$ (see Fig. 1) is also detected as a minimum of both $\mathrm{a}^{*}$ and $\mathrm{b}^{*}$ values when working with CIELab coordinates, and that this phenomenon is virtually absent in the case of adenosine solutions, also in accordance with Fig. 1.

In order to analyze those color changes, the two-state model was used as a first approximation. In this model, the change of an observed property $P$ is employed in order to obtain a binding constant $K$ by taking into account the value of the property for the free and bound substrate; in this case, the "free" parameter contribution corresponds to $\mathrm{a}^{*}$ and $\mathrm{b}^{*}$ values for non aggregated nanoparticles, and the "bound" to those corresponding to the biomolecule-AuNPs complex: ${ }^{25}$ to this end, a simplified reaction scheme, that assumes no preferential binding of the biomolecule to specific zones on the surface of the nanoparticle must be used; in other words, all binding sites on the nanoparticle are assumed to be equivalent: 

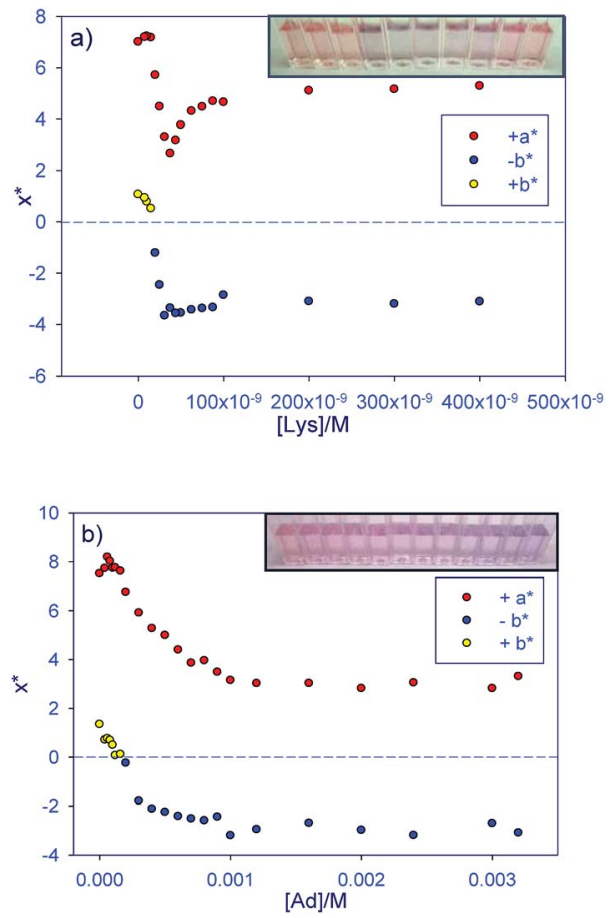

Fig. 2 (a) $\mathrm{a}^{*}$ and $\mathrm{b}^{*}$ parameters for a series of [AuNPs] $=2.8 \times 10^{-10} \mathrm{M}$ solutions containing lysozyme ([Lys] $=1 \times 10^{-9} \mathrm{M}$ to $4 \times 10^{-7} \mathrm{M}$ ). Points corresponding to $b^{*}$ measurements are colored in accordance to the tone expressed in the CIELab system determined by their positive or negative sign: positive $b^{*}$ values indicate a yellow tone, while negative $b^{*}$ values indicate a blue tone. Inset shows tonality changes with increasing lysozyme concentrations on a wider concentration range, as to better appreciate disaggregation effects: from left to right, [Lys] $=0 \mathrm{M}, 7.5 \times 10^{-10} \mathrm{M}, 2.5 \times 10^{-9} \mathrm{M}, 7.5 \times 10^{-9}$ $\mathrm{M}, 2.5 \times 10^{-8} \mathrm{M}, 7.5 \times 10^{-8} \mathrm{M}, 2.5 \times 10^{-7} \mathrm{M}, 2.5 \times 10^{-6} \mathrm{M}, 2.5 \times 10^{-5}$ $\mathrm{M}$ and $5 \times 10^{-4} \mathrm{M}$. (b) $\mathrm{a}^{*}$ and $\mathrm{b} *$ parameters for a series of [AuNPs] $=$ $2.8 \times 10^{-10} \mathrm{M}$ solutions containing adenosine ( $[\mathrm{Ad}]=2 \times 10^{-4}$ to $5 \times$ $10^{-3} \mathrm{M}$ ). Points corresponding to $b^{*}$ measurements are colored in accordance. Photograph shows tonality changes when increasing adenosine concentration from 0 to $3.2 \times 10^{-3} \mathrm{M}$.

$$
\begin{gathered}
\text { AuNPs }+ \text { substrate } \stackrel{K}{\leftrightarrow} \text { AuNPs } / \mathrm{S} \\
{[\text { AuNPs }]_{\text {free }}=\frac{[\mathrm{AuNPs}]_{\mathrm{total}}}{1+K[\mathrm{~S}]}} \\
{[\mathrm{AuNPs} / \mathrm{S}]=\frac{[\mathrm{AuNPs}]_{\mathrm{total}} K[\mathrm{~S}]}{1+K[\mathrm{~S}]}} \\
P_{\mathrm{obs}}=\frac{P_{\mathrm{AuNps}}+P_{\mathrm{AuNPs} / S} K[\mathrm{~S}]}{1+K[\mathrm{~S}]}
\end{gathered}
$$

where $P$ is the system property being determined by experimental procedures, and $\mathrm{S}$ the biomolecule acting as substrate.

Fig. 3 and $\mathrm{S} 4 \uparrow$ show the fit when using the two state model, by considering $a^{*}$ or $b^{*}$ as the observed property in eqn (3). From those fits, the two values of binding free energy obtained from $\mathrm{a}^{*}$ and $\mathrm{b}^{*}$ measurements are $-42.5 \mathrm{~kJ} \mathrm{~mol}^{-1}$ and $-45.2 \mathrm{~kJ}$ $\mathrm{mol}^{-1}$ for lysozyme, and $-18.4 \mathrm{~kJ} \mathrm{~mol}^{-1}$ and $-21.0 \mathrm{~kJ} \mathrm{~mol}^{-1}$ for adenosine.
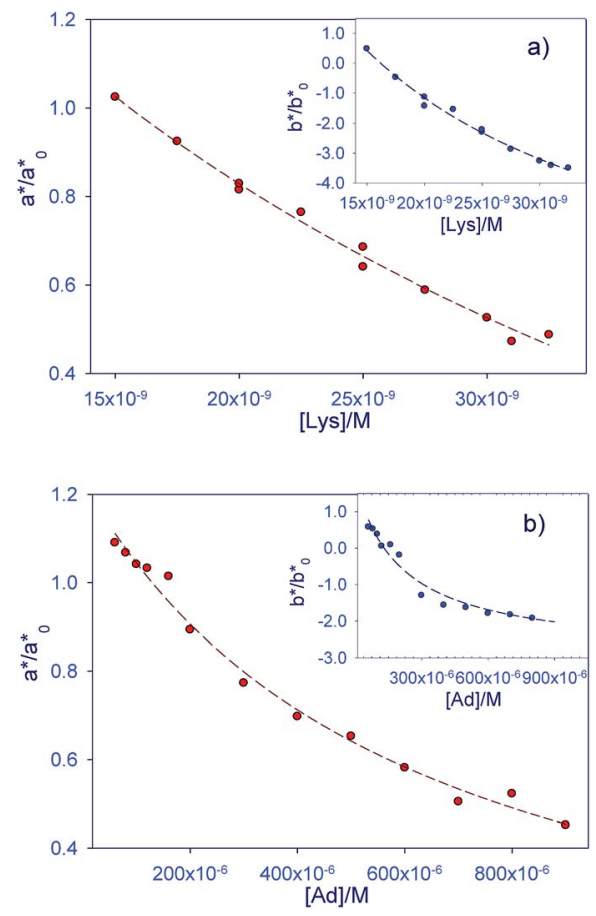

Fig. 3 (a) Two-state model fitting for normalized a* and b* (shown in the inset) parameters of AuNPs/lysozyme solutions on the aggregation-increasing zone ([AuNPs] $=2.8 \times 10^{-10} \mathrm{M}$; [Lys] $=0-3.75 \times 10^{-8}$ M) (b) two-state model fitting for $a^{*}$ and $b *$ parameters of AuNPs/ adenosine solutions $\left([\mathrm{AuNPs}]=2.8 \times 10^{-10} ;[\mathrm{Ad}]=0-9 \times 10^{-4} \mathrm{M}\right)$.

In order to confirm those results and test the effectiveness of the CIELab parameters for binding energy quantification, for each experimental absorbance spectra, the individual peaks corresponding to free and bound gold nanoparticles were determined by using a mathematical deconvolution procedure (Fig. 4). In this way, it is possible to split the experimental spectra and obtain the contribution of each individual band, allowing for the determination of their characteristic parameters and of the area corresponding to free and bound gold nanoparticles.

After performing the deconvolution, it was apparent that the AuNPs/S complex absorbance band both shifted to higher $\lambda_{\max }$

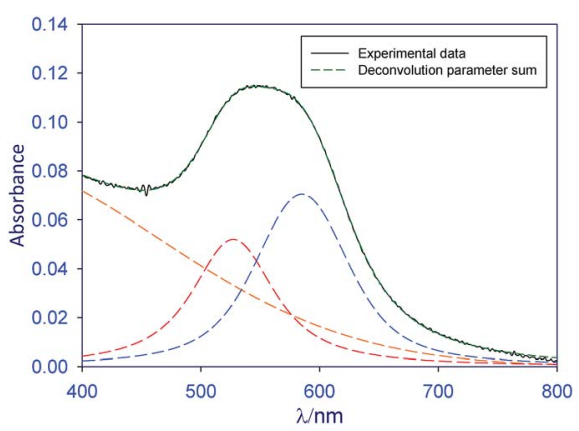

Fig. 4 Deconvolution fit for a AuNPs/Lys solution where [AuNPs] 2.8 $\times 10^{-10} \mathrm{M}$, [Lys] $=2.8 \times 10^{-8} \mathrm{M}$. The absorbance peaks corresponding to the free and aggregated nanoparticle fractions are marked in red and blue respectively. 
and broadened significantly with increasing substrate concentrations due to the aggregates' size distribution not being constant throughout the process. For adenosine, the aggregated absorbance band split into two different bands, which could be attributed to two distinct aggregated structures forming at the same time. Due to this fact, the normalized (area/area quotient) free AuNPs absorbance band was used for all fits.

The two-state model fit when using the normalized red peak area of the deconvolution fit (see Fig. 5 and $55 \dagger$ ) gives a $K$ value of $1.03 \times 10^{7} \mathrm{M}^{-1}$ for lysozyme, corresponding to a binding free energy of $-40.0 \mathrm{~kJ} \mathrm{~mol}^{-1}$. This data is clearly similar to the pair obtained when CIELab parameters are used (see Table 1). For adenosine, the value was $K=8.1 \times 10^{3} \mathrm{M}^{-1}$, which gives a binding free energy of $-22.3 \mathrm{~kJ} \mathrm{~mol}^{-1}$, also in accordance with CIELab-obtained $\Delta G^{0}$ values.

In order to ratify the proposed procedure, the Benesi-Hildebrand equation has also been used as an alternate data fitting method. ${ }^{26}$

$$
\frac{[\mathrm{AuNPs}]_{0}}{P}=\frac{1}{K \varepsilon[\mathrm{S}]_{0}}+\frac{1}{\varepsilon}
$$

where $K$ is the formation constant of the complex, $P$ is the property being measured, $\varepsilon$ a coefficient that depends on $P$, and $\mathrm{S}$ stands for the biomolecule acting as substrate. The BenesiHildebrand equation assumes that a direct, first order correlation exists between $P$ and the concentration of the complex; in its usual formulation, which identifies $P$ with the absorbance of the product at a fixed wavelength, this relationship is explained by way of the Lambert-Beer equation and $\varepsilon$ corresponds to the
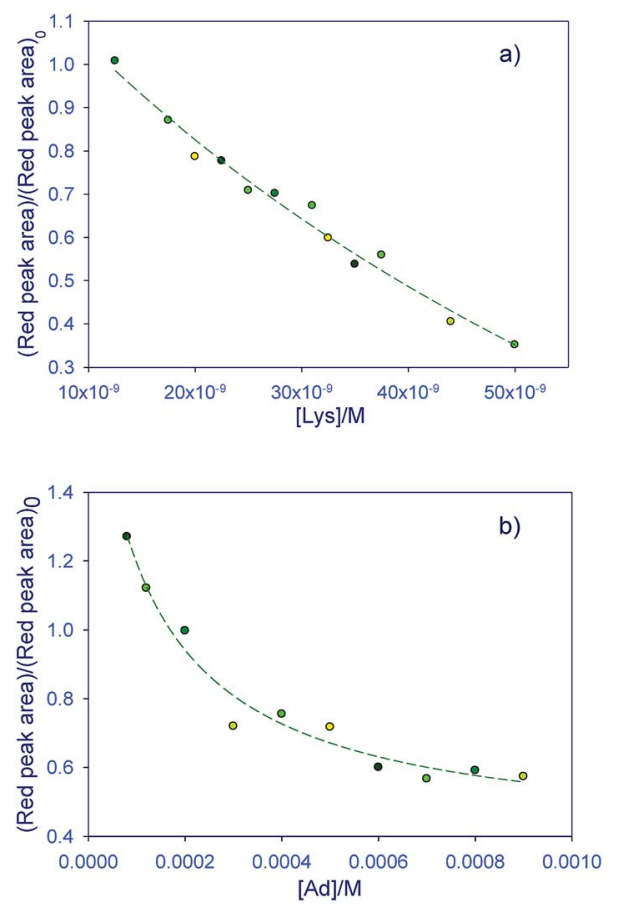

Fig. 5 Two-state model fit for the red (non-aggregated) deconvolution peak area for (a) a series of AuNPs/lysozyme solutions ranging from [Lys] $=1.3 \times 10^{-8}$ to $5 \times 10^{-8} \mathrm{M}$ and (b) a series of AuNPs/ adenosine solutions ranging from $[\mathrm{Ad}]=0$ to $9 \times 10^{-4} \mathrm{M}$.
Table 1 Binding free energy corresponding to the parameters obtained from deconvolution and CIELab value fitting

\begin{tabular}{|c|c|}
\hline Lysozyme & $\Delta G^{0} / \mathrm{kJ} \mathrm{mol}^{-1}$ \\
\hline Two-state model (deconvolution values) & $-40.0 \pm 1.2$ \\
\hline Two-state model (CIELab values) & $\begin{array}{l}-42.5 \pm 1.6\left(\mathrm{a}^{*}\right) \\
-45.2 \pm 1.7\left(\mathrm{~b}^{*}\right)\end{array}$ \\
\hline $\begin{array}{l}\text { Benesi-Hildebrand method (deconvolution } \\
\text { values) }\end{array}$ & $-43.8 \pm 0.2$ \\
\hline Benesi-Hildebrand method (CIELab values) & $\begin{array}{l}-44.4 \pm 0.5\left(\mathrm{a}^{*}\right) \\
-42.3 \pm 0.4\left(\mathrm{~b}^{*}\right)\end{array}$ \\
\hline Adenosine & $\Delta G^{0} / \mathrm{kJ} \mathrm{mol}^{-1}$ \\
\hline Two-state model (deconvolution values) & $-22.3 \pm 1.3$ \\
\hline Two-state model (CIELab values) & $\begin{array}{l}-18.4 \pm 0.7\left(\mathrm{a}^{*}\right) \\
-21.0 \pm 1.2\left(\mathrm{~b}^{*}\right)\end{array}$ \\
\hline $\begin{array}{l}\text { Benesi-Hildebrand method (deconvolution } \\
\text { values) }\end{array}$ & $-21.7 \pm 0.5$ \\
\hline Benesi-Hildebrand method (CIELab values) & $\begin{array}{l}-20.6 \pm 0.2\left(\mathrm{a}^{*}\right) \\
-21.5 \pm 0.6\left(\mathrm{~b}^{*}\right)\end{array}$ \\
\hline Lysine & $\Delta G^{0} / \mathrm{kJ} \mathrm{mol}{ }^{-1}$ \\
\hline Two-state model (deconvolution values) & $-11.8 \pm 0.8$ \\
\hline Two-state model (CIELab values) & $\begin{array}{l}-11.8 \pm 0.7\left(\mathrm{a}^{*}\right) \\
-13.9 \pm 0.7\left(\mathrm{~b}^{*}\right)\end{array}$ \\
\hline $\begin{array}{l}\text { Benesi-Hildebrand method (deconvolution } \\
\text { values) }\end{array}$ & $-12.1 \pm 0.4$ \\
\hline Benesi-Hildebrand method (CIELab values) & $\begin{array}{l}-13.4 \pm 0.2\left(\mathrm{a}^{*}\right) \\
-10.7 \pm 0.03\left(\mathrm{~b}^{*}\right)\end{array}$ \\
\hline Thiourea & $\Delta G^{0} / \mathrm{kJ} \mathrm{mol}^{-1}$ \\
\hline Two-state model (deconvolution values) & $-34.7 \pm 0.5$ \\
\hline Two-state model (CIELab values) & $\begin{array}{l}-37.2 \pm 0.6\left(a^{*}\right) \\
-36.2 \pm 0.6\left(b^{*}\right)\end{array}$ \\
\hline $\begin{array}{l}\text { Benesi-Hildebrand method (deconvolution } \\
\text { values) }\end{array}$ & $-36.9 \pm 0.1$ \\
\hline Benesi-Hildebrand method (CIELab values) & $\begin{array}{l}-39.6 \pm 0.1\left(\mathrm{a}^{*}\right) \\
-34.5 \pm 0.2\left(\mathrm{~b}^{*}\right)\end{array}$ \\
\hline
\end{tabular}

molar extinction coefficient of the complex. However, the previously explained phenomena of red-shifting and broadening that affect the AuNPs/receptor complex absorbance band makes those values unreliable for the determination of $K$, since the equation assumes that the complex band remains stable both in shape and in maxima position, as is common in most inorganic complexes. Again, those issues can be avoided by using parameters related to the free AuNPs absorbance band; the deconvolution procedures allow for the use of its normalized area instead of absorbance intensity, greatly improving the sensitivity of the method. It is important to note, however, that the use of area values for those calculations makes the $\varepsilon$ parameter not correspond to the extinction coefficient, since this coefficient is linked to absorbance at a fixed wavelength. However, the experimental straight line relationship (see Fig. 6 and $\mathrm{S}^{\dagger} \dagger$ ) that was found confirms that $\varepsilon$ remains constant when using the normalized peak area in order to measure $K$ or, in other words, that the free AuNPs peak area is directly linked to the concentration of aggregated AuNPs in solution. In addition, when $\mathrm{a}^{*}$ and $\mathrm{b}^{*}$ values were used as the property $P$, a linear 
relationship zone was also found both for lysozyme and for adenosine (see Fig. 7 and $\mathrm{S} 7 \dagger$ ). This fact is not surprising, since it is well known that for nanoparticle systems, color is closely related to the state of the system; and, as demonstrated before (see Fig. 2), a relationship can be found between the values of those two parameters and the concentration of aggregated nanoparticles.

Lysozyme contains six tryptophan (Trp) residues, which present appreciable fluorescence and in principle should allow for the use of molecular fluorescence techniques to monitor AuNPs/lysozyme interactions. As can be seen from fluorescence intensity measurements of the protein in the presence of different AuNPs concentrations (see Fig. 8), the fluorescence of lysozyme is quenched in the presence of AuNPs, a fact that allows for the use of the two-state model in order to obtain fluorescence binding constants.

From the fit, a value of $1.6 \times 10^{9} \mathrm{M}^{-1}$ is obtained, which corresponds to a binding free energy of $-52.5 \mathrm{~kJ} \mathrm{~mol}^{-1}$. However, the fit of the experimental data is not good. Clearly, experimental data corresponding to the highest concentrations of gold are out of adjustment. This situation can arise due to different causes, for example coupled static and dynamic quenching, a secondary reaction process or an inner filter effect. $^{27,28}$

A Stern-Volmer plot of [AuNPs] versus $I_{0} / I$ (Fig. 9) shows positive deviations of linearity for the higher [AuNPs] concentrations; this phenomenon indicates higher experimental quenching intensities than those predicted by a one-constant linear relationship, and can be indicative of the presence of
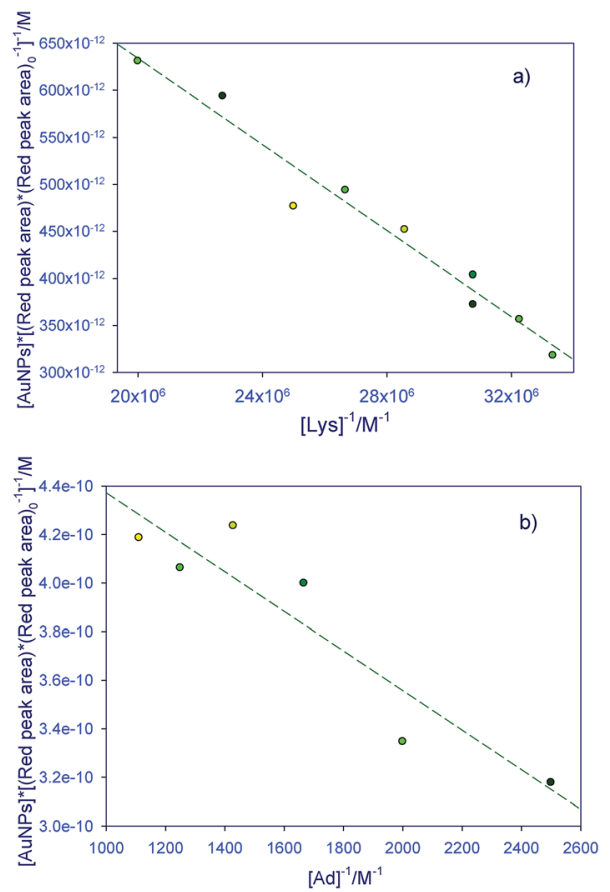

Fig. 6 Benesi-Hildebrand fit for the normalized red peak area obtained from deconvolution procedures for (a) a series of AuNPs/lysozyme solutions ranging from [Lys] $=3 \times 10^{-8}$ to $6.25 \times 10^{-8} \mathrm{M}$ and (b) AuNPs/ adenosine solutions ranging from $[\mathrm{Ad}]=8 \times 10^{-5}$ to $5 \times 10^{-4} \mathrm{M}$.
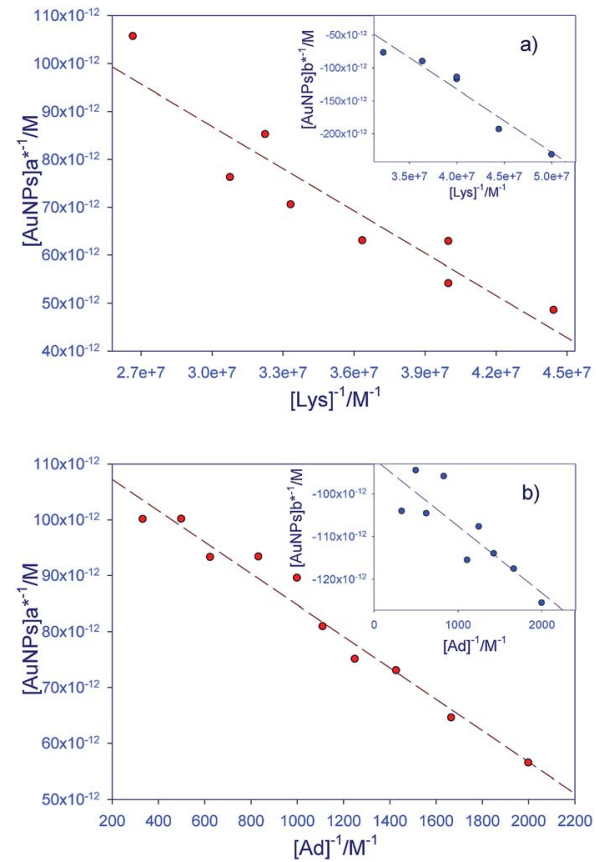

Fig. 7 Benesi-Hildebrand fit for $a *$ and $b *$ (shown on inset) for a series of AuNPs solutions ranging from (a) [Lys] $=2 \times 10^{-8}$ to $3.75 \times 10^{-8} \mathrm{M}$ and (b) $[\mathrm{Ad}]=5 \times 10^{-4}$ to $3 \times 10^{-3} \mathrm{M}$.

mixed static and dynamic quenching effects. However, when fitting to a second-grade Stern-Volmer equation no logical values were found for either constant, which can signal the influence of an external, non-constant factor into the experimental measurements. It is remarkable to note that the higher deviations from linearity are found for lower lysozyme concentrations, which show lesser fluorescence values and higher [AuNPs]/[Lys] ratios. AuNPs have been reported to absorb protein fluorescence when the fluorophore's maximum emission wavelength is close to or overlapping with that of the SPR of the nanoparticle. ${ }^{27}$ On the other hand, fluorescence light can be scattered by the metal core of the nanoparticle, reducing the fluorescence signal; this effect is particularly notable when the protein emission maximum is located near the blue zone of the spectrum, since shorter wavelengths are more susceptible to light scattering effects. Whatever the situation it is clear that the

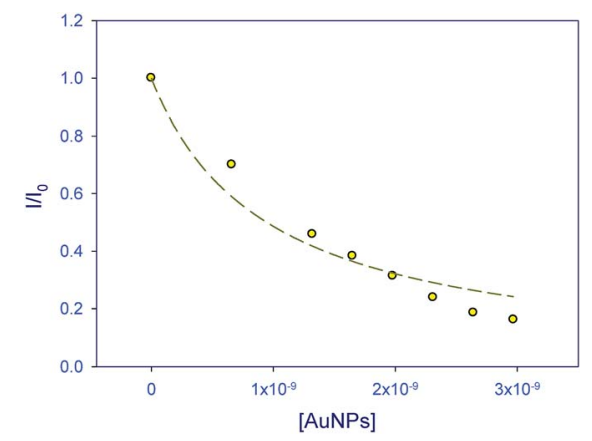

Fig. 8 Two-state model fitting for experimental lysozyme fluorescence quenching data for $\left[\right.$ Lys] $=5 \times 10^{-6} \mathrm{M}$. 


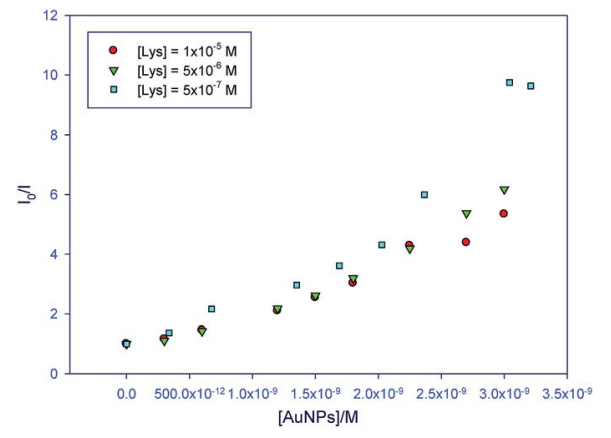

Fig. 9 Stern-Volmer representation at [Lys] $=1 \times 10^{-5} \mathrm{M}, 5 \times 10^{-6} \mathrm{M}$ and $5 \times 10^{-7} \mathrm{M}$.

influence of physical effects on lysozyme fluorescence measurements makes quenching curves not representative of the binding of lysozyme to AuNPs. It is also important to note that ionic strength markedly influences fluorescence measurements, and in this sense it is not easy to use fluorimetric techniques when comparing data a priori corresponding to the same system. It is absolutely necessary for AuNPs employed on different measurements to have exactly the same citrate concentration, and of course same diameter nanoparticles should be employed (taking into account the ever-present size distribution of nanoparticle systems). It is remarkable to note that binding energies obtained from color and deconvolution fits are all inferior to the one from fluorescence quenching measurements; this fact further proves the point that positive deviations of fluorescence at high [AuNPs] cause the emissioncalculated binding energy to be greater than expected.

\section{Experimental section}

\section{Reagents and nanoparticle characterization}

All commercial chemicals mentioned from here on were of Anal. R. Grade. Lysozyme (from chicken egg white) [L6876] and adenosine [A9251] were purchased from Sigma-Aldrich, L-lysine monohydrochloride from Merck [1057000100] and thiourea from VWR Chemicals [28615.231]. All reactants were used without further purification. Citrate-capped gold nanoparticles were prepared by direct reduction of $\mathrm{HAuCl}_{4}$ salts [SigmaAldrich, 520918] with sodium citrate [Riedel-de Haën, 32 320] using heat and continuous magnetical stirring in aqueous media. ${ }^{29}$ Characterization of the resulting colloid was carried out by TEM measurements and the size and shape of the AuNPs were analyzed by using ImageJ software over a sample set of over 300 nanoparticles. The mean diameter of the synthesized citrate-AuNPs was found to be $17.3 \mathrm{~nm}$ with a dispersion coefficient of $1.1 \mathrm{~nm}$, which represents less than $7 \%$ of the mean particle size, meaning that the colloid can be considered monodisperse (see Fig. S1†).

The circularity of the sample particles, defined as:

$$
\text { Circularity }=4 \pi \times \frac{\text { area }}{\text { perimeter }^{2}}
$$

and which ranges from 0 to 1 (total circularity) was over 0.835 in all measured particles, with a mean value of 0.916 , which implies near-perfect sphericity of the AuNPs.

\section{Methods and instrumentation}

Fluorescence spectra were obtained by using a Hitachi F2500 fluorescence spectrophotometer, working at fixed [Lys] concentrations and $\left[\right.$ AuNPs] $=0$ to $3 \times 10^{-9} \mathrm{M}$. Transmittance spectra were recorded by using a Cary500 spectrophotometer at $298.3 \mathrm{~K}$ from 400 to $800 \mathrm{~nm}$, at AuNPs $=2.8 \times 10^{-10} \mathrm{M}$ and varying concentrations of lysozyme and adenosine.

TEM measurements. For TEM examinations, a single drop $(10 \mu \mathrm{l})$ of the aqueous solution of gold nanoparticles was placed on a copper grid coated with a carbon film. The grid was left to dry in air for several hours at room temperature. TEM analysis for synthesis characterization was carried out in a Hitachi CM 200 electron microscope working at $200 \mathrm{kV}$.

CIELab data obtention. Conversion from transmittance measurements to CIELab chromaticity system parameters was performed by using CIE standard equations to convert experimental measurements to XYZ chromaticity values, and then to $\mathrm{L} * \mathrm{a} * \mathrm{~b} * \mathbf{3 0}$ (see ESI $\dagger$ ).

Deconvolution procedure. Deconvolution of experimental absorbance spectra was done with the help of Fityk deconvolution software. ${ }^{31}$ In all cases, spectra were fit to Voigt functions in order to account for the non-gaussian shape of the surface plasmon absorbance band. The deconvolution model used a free AuNPs absorbance band centered at around $521 \mathrm{~nm}$; one or two bound AuNPs absorbance bands over $530 \mathrm{~nm}$, which correspond to aggregated nanoparticles of different size distributions; and a broad band that was fixed at $300 \mathrm{~nm}$ and accounted for both residual $\mathrm{Au}^{3+}$ salts absorbance ${ }^{32}$ and for light scattering phenomena caused by the presence of gold nanoparticles in suspension at smaller wavelengths.

\section{Conclusions}

CIELab measurements offer a powerful, fast, and direct way to determine the free energy of binding of a nanoparticle/ biomolecule system. It also has a more than acceptable accuracy: less than $10 \%$ error was found for all tested biomolecules. Moreover, since this detection method is based on the strong colors of gold nanoparticles, it can be effectively used for the determination of binding energies of non-colored, nonfluorescent reactants of biological interest such as nucleosides or amino acids.

CIELab measurements have additional advantages for nanoparticle systems in that they are not dependent of the maximum absorbance peaks, whose position and intensity greatly depend of the type, size and proportion between different types of aggregates formed and so tends to show questionable reproducibility. Since all aggregates cause the blue tint to appear, and due to the red absorbance zone of the visible spectrum being quite broad, the blue color does not differ on an appreciable basis between different aggregate structures and proportions, making CIELab parameters useful for 
determination of the binding parameters with independence of the aggregated structure that results from the process. It can also be applied without the need for a previous mathematical processing of the spectra, unlike deconvolution procedures where the mathematical function types have to be carefully chosen for each system in order to assure a good fit to the experimental data. In fact, CIELab data can be directly obtained from experimental measurements with the use of appropriate colorimetric equipment. To our knowledge, this is the first time CIELab procedures have been applied as shown for the determination of binding free energies of biomolecules to gold nanoparticles. The fact that even otherwise non-measurable nanoparticle/biomolecule systems can be observed with this method causes it to stand up strongly against more traditional measurement procedures, making it worth of seeing more development and refining in the near-future.

\section{Acknowledgements}

We thank Dr F. Varela (CITIUS, University of Seville) for his assistance at obtaining TEM images. We thank University of Sevilla for a grant of the V Programa Propio.

\section{Notes and references}

1 R. Elghanian, J. J. Storhoff, R. C. Mucic, R. L. Letsinger and C. A. Mirkin, Science, 1997, 277, 1078-1081.

2 O. Kadioglu, A. H. Malczyk, H. J. Greten and T. Efferth, Invest. New Drugs, 2015, 33, 513-520.

3 L. Gong, Z. Zhao, Y.-F. Lv, S.-Y. Huan, T. Fu, X.-B. Zhang, G.-L. Shen and R.-Q. Yu, Chem. Commun., 2015, 51, 979-995.

4 J. Zhang, Y. Tang, L. Teng, M. Lu and D. Tang, Biosens. Bioelectron., 2015, 68, 232-238.

5 A. P. Echavarría, J. Pagán and A. Ibarz, Food Sci. Technol. Int., 2014, 20, 119-126.

6 C. Niamnuy, S. Devahastin, S. Soponronnarit and G. S. Vijaya Raghavan, J. Food Eng., 2008, 87, 591-600.

7 K. C. Kwok, D. B. MacDougall and K. Niranjan, J. Food Eng., 1999, 40, 15-20.

8 M. Matsuo, K. Umemura and S. Kawai, J. Wood Sci., 2011, 58, 113-119.

9 M. Torrell, P. Machado, L. Cunha, N. M. Figueiredo, J. C. Oliveira, C. Louro and F. Vaz, Surf. Coat. Technol., 2010, 204, 1569-1575.

10 R. E. Noble and H. H. Fudenberg, Blood, 1967, 30, 465-473.
11 S. S. Levinson, R. J. Elin and L. Yam, Clin. Chem., 2002, 48, 1131-1132.

12 J. F. Harrison, G. S. Lunt, P. Scott and J. D. Blainey, Lancet, 1968, 1, 371-375.

13 M. Klockars, S. Reitamo, T. Weber and Y. Kerttula, Acta Med. Scand., 1978, 203, 71-74.

14 S. M. Miller, A. Kato and S. J. Nakai, J. Agric. Food Chem., 1982, 30, 1127-1132.

15 Y. U. Moon, R. A. Curtis, C. O. Anderson, H. W. Blanch and J. M. J. Prausnitz, J. Solution Chem., 2000, 26, 699-717.

16 L. M. Demers, M. Östblom, H. Zhang, N.-H. Jang, B. Liedberg and C. A. Mirkin, J. Am. Chem. Soc., 2002, 124, 11248-11249.

17 S. Basu, S. Jana, S. Pande and T. Pal, J. Colloid Interface Sci., 2008, 321, 288-293.

18 H. Kimura-Suda, D. Y. Petrovykh, M. J. Tarlov and L. J. Whitman, J. Am. Chem. Soc., 2003, 125, 9014-9015.

19 S. Piana and A. Bilic, J. Phys. Chem. B, 2006, 110, 2346723471.

20 J. Yang, B.-K. Pong, J. Y. Lee and H.-P. Too, J. Inorg. Biochem., 2007, 101, 824-830.

21 J. J. Storhoff, R. Elghanian, C. A. Mirkin and R. L. Letsinger, Langmuir, 2002, 18, 6666-6670.

22 S. Udenfriend and P. Zaltzman, Anal. Biochem., 1962, 3, 4959.

23 W. B. Knighton, G. Giskaas and P. R. Caills, J. Phys. Chem. B, 1982, 86, 49-55.

24 Y.-L. Cao, Y. Li, F. Zhang, J.-Z. Huo and X.-J. Zhao, Anal. Methods, 2015, 7, 4927-4933.

25 R. Prado-Gotor, E. Grueso, P. Perez-Tejeda, A. Jimenez-Ruiz and J. M. Carnerero, Soft Matter, 2014, 10, 8482-8488.

26 H. A. Benesi and J. H. Hildebrand, J. Am. Chem. Soc., 1949, 71, 2703-2707.

27 J. A. Yang, B. J. Johnson, S. Wu, W. S. Woods, J. M. George and C. J. Murphy, Langmuir, 2013, 29, 4603-4615.

28 S. P. Boulos, T. A. Davis, J. A. Yang, S. E. Lohse, A. M. Alkilany, L. A. Holland and C. J. Murphy, Langmuir, 2013, 29, 14984-14996.

29 X. Wang, Y. Xu, X. Xu, K. Hu, M. Xiang, L. Li, F. Liu and N. Li, Talanta, 2010, 82, 693-697.

30 OIV, Compendium of International Methods of Wine and Must Analysis, Paris (France), 2014.

31 M. Wojdyr, J. Appl. Crystallogr., 2010, 43, 1126-1128.

32 C.-S. Yang, M.-S. Shian and F.-Y. Chang, New J. Chem., 2006, 30, 729-735. 